\title{
Verzeichnis der Wicklungs-Schaltbilder
}

I. 2polige Ein- und Zweischichten-Dreiphasenwicklungen (Gegenüberstellung verschiedener Wicklungsarten) . . . . . . . . 21-24

II. Ausgleichverbindungen bei Gleichstrom-Ankerwicklungen . . . . . 149

1II. Parallelschalten 4 poliger Nebenschlußwicklungen bei Gleichstrommaschinen . . . . . . . . . . . . . . . . . . 153

IV. Dreiphasen-Einschicht-Bruchlochwicklungen

4 polig 18 Nuten, Abb. Nr. 122. . . . . . . . . . . . . . 160

30 Nuten, Abb. Nr. 124. . . . . . . . . . . . 161

27 Nuten, Abb. Nr. 125. . . . . . . . . . . . . . . . . 162

42 Nuten, Abb. Nr. 132. . . . . . . . . . . . 165

54 Nuten, Abb. Nr. 139. . . . . . . . . . . . . . . 168

6 polig 24 Nuten, Abb. Nr. 202. . . . . . . . . . . . . 247

30 Nuten, Abb. Nr. 126. . . . . . . . . . . . . . . . 163

39 Nuten, Abb. Nr. 130. . . . . . . . . . . . 164

45 Nuten, Abb. Nr. 133. . . . . . . . . . . . . . 166

48 Nuten, Abb. Nr. 136. . . . . . . . . . . . . . . . . 167

8 polig 30 Nuten, Abb. Nr. 127. . . . . . . . . . . . 163

36 Nuten, Abb. Nr. 128. . . . . . . . . . . . . . . . . 163

36 Nuten, Abb. Nr. 208. . . . . . . . . . . . . . 250

42 Nuten, Abb. Nr. 131. . . . . . . . . . . . 164

48 Nuten, Abb. Nr. 137. . . . . . . . . . . . . . . . . 167

54 Nuten, Abb. Nr. 140. . . . . . . . . . . . . . . 169

54 Nuten, Abb. Nr. 169. . . . . . . . . . . 217

60 Nuten, Abb. Nr. 143. . . . . . . . . . . . . . 170

66 Nuten, Abb. Nr. 157. . . . . . . . . . . . . 187

84 Nuten, Abb. Nr. 144 u. 147 . . . . . . . . . 171 11.173

90 Nuten, Abb. Nr. 158. . . . . . . . . . . . . . . 188

90 Nuten, Abb. Nr. 160. . . . . . . . . . . . . 190

10 polig 18 Nuten, Abb. Nr. 123. . . . . . . . . . . 161

24 Nuten, Abb. Nr. 121. . . . . . . . . . . . 158

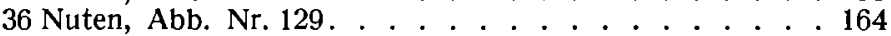

45 Nuten, Abb. Nr. 134 u. 135 . . . . . . . . . . . .166-167

48 Nuten, Abb. Nr. 138. . . . . . . . . . . . 168

54 Nuten, Abb. Nr. 141. . . . . . . . . . . . . . . . . 169

72 Nuten, Abb. Nr. 145. . . . . . . . . . . . . . 172

96 Nuten, Abb. Nr. 159. . . . . . . . . . . . . 189

12 polig 54 Nuten, Abb. Nr. 142. . . . . . . . . . . . . 169

60 Nuten, Abb. Nr. 146, 155, 156 . . . . . . . $173,185,186$

V. Dreiphasen-Zweischichten-Bruchlochwicklungen

4 polig 18 Nuten, Abb. Nr. 233. . . . . . . . . . . . . 273

27 Nuten, Abb. Nr. 236. . . . . . . . . . . 275 
30 Nuten, Abb. Nr. 237. . . . . . . . . . . 276

42 Nuten, Abb. Nr. 240. . . . . . . . . . . . . . . 279

6 polig 24 Nuten, Abb. Nr. 243. . . . . . . . . . . . . 281

27 Nuten, Abb. Nr. 152. . . . . . . . . . . . . . 183

48 Nuten, Abb. Nr. 136. . . . . . . . . . . 167

8 polig 30 Nuten, Abb. Nr. 247. . . . . . . . . . . . . . 283

36 Nuten, Abb. Nr. 248. . . . . . . . . . . . . . 283

42 Nuten, Abb. Nr. 249. . . . . . . . . . . . . . . . 284

54 Nuten, Abb. Nr. 251. . . . . . . . . . . . . . . . . 285

60 Nuten, Abb. Nr. 252. . . . . . . . . . . . 286

60 Nuten, Abb. Nr. 253. . . . . . . . . . . . . 287

66 Nuten, Abb. Nr. 254. . . . . . . . . . . . . . . 288

78 Nuten, Abb. Nr. 255. . . . . . . . . . . . . . . . . 289

VI. Dreiphasen-Einschicht-Ganzlochwicklungen

2 polig 12 Nuten, Abb. Nr. 197. . . . . . . . . . . . . 244

18 Nuten, Abb. Nr. 199. . . . . . . . . . . . . 245

24 Nuten, Abb. Nr. 200. . . . . . . . . . . . . . . . 246

36 Nuten, Abb. Nr. 203. . . . . . . . . . . . . . . 247

36 Nuten, Abb. Nr.204. . . . . . . . . . . . . . . . . 248

4 polig 12 Nuten, Abb. Nr. 198. . . . . . . . . . . . . . . 245

24 Nuten, Abb. Nr. 201. . . . . . . . . . . . . . . . . 246

36 Nuten, Abb. Nr. 206. . . . . . . . . . . . . . . . . 249

48 Nuten, Abb. Nr. 212. . . . . . . . . . . . . . . . 252

6 polig 36 Nuten, Abb. Nr. 207. . . . . . . . . . . . . . 249

8 polig 48 Nuten, Abb. Nr. 209. . . . . . . . . . . . 250

72 Nuten, Abb. Nr.210. . . . . . . . . . . 251

72 Nuten, Abb. Nr. 211. . . . . . . . . . . 251

10 polig 60 Nuten, Abb. Nr. 205. . . . . . . . . . . 248

VII. Dreiphasen-Zweischichten-Ganzlochwicklungen

2 polig 12 Nuten, Abb. Nr. 228. . . . . . . . . . . . . . 272

18 Nuten, Abb. Nr. 229. . . . . . . . . . . . 272

24 Nuten, Abb. Nr. 230 . . . . . . . . . . . . . . . 272

36 Nuten, Abb. Nr. 232. . . . . . . . . . . . 273

4 polig 24 Nuten, Abb. Nr. 231. . . . . . . . . . . . . 272

36 Nuten, Abb. Nr. 224 (Schaltplan) . . . . . . . . . . 264

36 Nuten, Abb. Nr. 241............. . . . 280

36 Nuten, Abb. Nr. 238. . . . . . . . . . . . . . . 277

36 Nuten, Abb. Nr. 239 (2 Stränge parallel). . . . . . . . 278

48 Nuten, Abb. Nr. 242. . . . . . . . . . . . 280

6polig 36 Nuten, Abb. Nr. 244. . . . . . . . . . . . 281

54 Nuten, Abb. Nr. 245. . . . . . . . . . . . . . . . . 282

72 Nuten, Abb. Nr. 246. . . . . . . . . . . . . 282

8 polig 48 Nuten, Abb. Nr. 250. . . . . . . . . . . . 284

72 Nuten, Abb. Nr. 257. . . . . . . . . . . . 290

VIII. Dreiphasen-Zweischichten-Ganzlochwicklungen

mit Spulen gleicher oder oder ungl. Weite, für alle Nutenzahlen und

Wickelschritte. Vereinfachte Darstellung in 3 Wicklungssträngen getrennt, für Serien- und Parallelschaltung je Strang.

2 polig je Phase 2 Spulen in Serie, Abb. Nr. 258 . . . . . . . . 297

je Phase 2 Spulen parallel, Ahb. Nr. 259 . . . . . . . 298 
4 polig je Phase 4 Spulen in Serie, Abb. Nr. 260 . . . . . . . 299

je Phase 2 Spulen parallel, Abb. Nr. 261 . . . . . . . . 300

je Phase 4 Spulen parallel, Abb. Nr. 262 . . . . . . . 301

6 polig je Phase 6 Spulen in Serie, Abb. Nr. 263 . . . . . . 302

je Phase 2 Spulen parallel, Abb. Nr. 264 . . . . . . . 303

je Phase 3 Spulen parallel, Abb. Nr. 265 . . . . . . . 304

je Phase 6 Spulen parallel, Abb. Nr. 266 . . . . . . . 305

8 polig je Phase 8 Spulen in Serie, Abb. Nr. 225 . . . . . . 268

je Phase 2 Spulen parallel, Abb. Nr. 267 . . . . . . . . 306

je Phase 4 Spulen parallel, Abb. Nr. 268 . . . . . . . 307

je Phase 8 Spulen parallel, Abb. Nr. 269 . . . . . . 308

IX. Polumschaltbare Dreiphasenwicklungen

4/2polig, 24 Nuten, Einsch. Abb. Nr. 278 . . . . . 322

4/2 polig, 24 Nuten, Zweisch. Abb. Nr. 285 . . . . . . 329

4/2 polig, 24 Nuten, Sp. Gl. W. Abb. Nr. 286 . . . . 329

4/2 polig, $\quad 36$ Nuten, Zweisch. Abb. Nr. 287 (Dahlander) 330

4/2 polig, 48 Nuten, Zweisch. Abb. Nr. 291 (Dahlander) 333

6/4 polig, 36 Nuten, Zweisch. Abb. Nr. 295 . . . . 337

6/4/2 polig, 48 Nuten, Einsch. Abb. Nr. $301 . . . . .343$

6/4/2 polig, 54 Nuten, Zweisch. Abb. Nr. 277 . . . . . 321

8/4 polig, 36 Nuten, Zweisch. Abb. Nr. 288 . . . . . . 331

8/4 polig, $\quad 48$ Nuten, Zweisch. Abb. Nr. 289 . . . . . 331

8/4 polig, $\quad 36$ Nuten, Zweisch. St. Dreieck Abb. Nr. 290 . 332

8/4 polig, 48 Nuten, Einsch. Abb. Nr. 279 (Dahlander) 323

8/6 polig, 36 Nuten, Einsch. Abb. Nr. 296 . . . . . 338

8/4/2 polig, 48 Nuten, Einsch. Abb. Nr. 299 . . . . . . 341

8/6/4 polig, 36 Nuten, Zweisch. Abb. Nr. 300 . . . . . 342

8/6/4 polig, 48 Nuten, Zweisch. Abb. Nr. 303 . . . . . . 345

8/6/4/2 polig, 36 Nuten, Zweisch. Abb. Nr. 293 . . . . . 335

12/6 polig, 60 Nuten, Einsch. Abb. Nr. 280 . . . . . . 324

12/6 polig, 60 Nuten, Einsch. Abb. Nr. 280 . . . . . . 324

12/ôpolig, 36 Nuten, Zweisch. Abb. Nr. $292 . . . .334$

12/6 polig, 72 Nuten, Einsch. Abb. Nr. $281 . . . . .325$

12/6 polig, 72 Nuten, Zweisch. Abb. Nr. 294 . . . . . 336

12/8/6/4 polig, 72 Nuten, Zweisch. Abb. Nr. 284 ..... . 328

IX. a) Polumschaltbare Dahlander-Wicklungen in vereinfachter Darstellung, $Z$ weischichtenwicklungen.

4/2polig für alle Nutenzahlen u. Wickelschritte, Abb. Nr.270 309

$8 / 4$ polig für alle Nutenzahlen u. Wickelschritte, Abb. Nr.271 310

$12 / 6$ polig für alle Nutenzahlen u. Wickelschritte, Abb. Nr.272 311

16/8polig für alle Wickelschritte u. Nutenzahlen, Abb. Nr.273 312

IX. b) Polumschalter 8/6/4 Pole SSW Weinert, Abb. Nr. 304 . . 346

Dreieck/Dreieck-Schaltung, 12 Klemmen, Abb. Nr. 302 . 344

Prinzipschaltungen für 2 u. 4 Drehzahlen, Abb. Nr. 282.326

Dahlander-Wicklung mit Polumschalter, Abb. Nr. 308 . . 349

Dahlander-Wicklung mit 3 Drehzahlen, Abb. Nr. 310 . 350

Dahlander-Wicklung mit 4 Drehzahlen, Abb. Nr. 283 . 327

X. Zweiphasen-Bruchlochwicklung.

4polig, 16 Nuten, Abb. Nr. 167 (Zweiphasen-Läuferwicklung) 203

4 polig, 36 Nuten, Abb. Nr. 161 . . . . . . . . . 191

4polig, 16 Nuten, Abb. Nr. 168(Zweiphasen-Formspulenwick-

lung) .................. 207 
XI. Einphasen-Wechselstrom-Wicklungen.

Abb. Nr. 330 bis Nr. $336 . . . . . . . . . .384$

Abb. Nr. 388 bis Nr. 409 . . . . . . . . . . . 445-453

XII. Sonderausführungen.

1. 6polige Dreiphasen-Zweischichtenwicklung, umschaltbar für die Netzspannungen 110, 125, 190,220, 330 und 380 Volt, Abb. Nr. 163............... 193

2. Umschaltbare Dreiphasenwicklung für das Spannungsverhältnis $2: 1$ (z. B. 440/220, 220/110 Volt), 4polige Zweischichtenwicklung, Abb. Nr. 164 . . . . . . . 200

3. 2-3- und 4-Lochspulen ungl. Weite, Abb. Nr. 165 . . . 201

4. Undurchführbare Parallelschaltung von Mehrfachspulen Abb. Nr. 166 . . . . . . . . . . . . . . . . . . . 201

5. Dreiphasen-Dreiebenen-Ganzlochwicklung, 48 Nuten, Abb. Nr. 214. . . . . . . . . . . . 254

6. Dreiphasen-Dreiebenen-Ganzlochwicklung für geteilte Ständer, 4 polig, 24 Nuten, Abb. Nr. 215 ....... 255

7. Universal-Schaltbild für 2/4/6- und 8 polige DreiphasenZweischichtenwicklungen Abb. Nr. 256. . . . . . . 290

8. 8polige Gleichstrom-Reihenparallelwicklung, 270 Nuten mit Ausgleichverbindungen Abb. 315 . . . . . . 356 